\title{
Genetic variation in the coagulation factor $V$ gene and risk of femoral head osteonecrosis
}

\author{
TAE-HO KIM ${ }^{1,2}$, SEUNG-HOON BAEK ${ }^{3}$, JEONG OK LIM $^{1}$, SANG-HAN LEE $^{4}$ and SHIN-YOON KIM ${ }^{2,3}$ \\ ${ }^{1}$ Biomedical Research Institute, Kyungpook National University Hospital; ${ }^{2}$ Skeletal Diseases Genome Research Center, \\ Kyungpook National University; ${ }^{3}$ Department of Orthopedic Surgery, Kyungpook National University Hospital, \\ Daegu 700-721; ${ }^{4}$ Department of Food Science and Biotechnology, Kyungpook National University, \\ Daegu 702-701, Republic of Korea
}

Received August 5, 2014; Accepted May 8, 2015

DOI: $10.3892 / \mathrm{mmr} .2015 .4000$

\begin{abstract}
Osteonecrosis of the femoral head (ONFH) is characterized by the death of the cellular portion of the femoral head due a reduction or disruption in the blood supply. Certain studies have implicated coagulation disorders, including thrombophilia and hypofibrinolysis, in the pathogenesis of ONFH. The factor $\mathrm{V}$ (F5) Leiden mutation has been suggested to be a genetic risk factor for venous thromboembolism and osteonecrosis in Caucasian individuals, however, this association remains controversial in other populations. The present study aimed to identify polymorphisms of the $F 5$ gene and performed a case-control study in a Korean population. The F5 gene was sequenced in 24 unrelated Korean individuals, and 16 polymorphisms were detected. A total of six polymorphisms were genotyped in 423 patients with ONFH and 348 control individuals. Analysis of the association between genotyped single nucleotide polymorphisms and haplotypes and with ONFH was performed. Comparison of the ONFH samples and the control individuals using logistic regression models revealed no statistically significant difference in the frequencies of the F5 polymorphisms and haplotypes. These findings suggested that F5 polymorphisms were not significant in the susceptibility to $\mathrm{ONFH}$ in the Korean population.
\end{abstract}

\section{Introduction}

Osteonecrosis of the femoral head (ONFH) is a debilitating bone disease, characterized by cellular death in the bone tissue

Correspondence to: Dr Sang-Han Lee, Department of Food Science and Biotechnology, Kyungpook National University, 80 Daehak-ro, Buk-gu, Daegu 702-701, Republic of Korea

E-mail: sang@knu.ac.kr

Dr Shin-Yoon Kim, Department of Orthopedic Surgery, Kyungpook National University Hospital, 130 Dongdeok-ro, Jung-gu, Daegu 700-721, Republic of Korea

E-mail: syukim@knu.ac.kr

Key words: osteonecrosis of the femoral head, factor V, polymorphism that can lead to collapse of the architectural bone structure and loss of joint function (1). Although several conditions, including steroids, alcoholism, coagulation defects, storage diseases, marrow-infiltrating diseases and certain autoimmune diseases, can increase the risk of ONFH, the pathogenesis remains to be fully elucidated (1-5). Several hypotheses regarding its pathogenesis, including intravascular coagulation (6,7), apoptosis of osteoblasts and osteocytes (8), and fatty necrosis of osteocytes (9), have been suggested. Among them, a vascular hypothesis is considered to be the most persuasive, which implicates a decrease in blood flow to the local femoral head in the pathogenesis of ONFH (10).

In support of this hypothesis, genetic mutations associated with coagulation abnormalities, including the factor $\mathrm{V}$ (F5) Leiden mutation (G1691A; Arg506Gln) and 5,10-methylene-tetrahydrofolate reductase gene polymorphism (C677T; Ala222Val), have been used to assess the role of genetics in the risk of ONFH (6,7). Of four studies investigating the role of the F5 Leiden mutation, three demonstrated a positive correlation with ONFH in Caucasian patients $(6,11,12)$. However, Lee et al $(13)$ reported no association between ONFH and increased thrombosis or impaired fibrinolysis in Korean patients with ONFH. Additionally, other studies have reported the absence of F5 Leiden and 20210A mutations in Koreans (14-16). It is possible that there are geographic and ethnic differences in the prevalence of these mutations.

Although the F5 Leiden mutation has not been observed in the Korean population, the $F 5$ gene is considered to be important in ONFH. Therefore, the present study performed extensive screening of the $F 5$ gene by direct sequencing to identify the polymorphisms and mutations, and examined the genetic association with ONFH risk in a Korean population.

\section{Patients and methods}

Patients. Unrelated patients, diagnosed with $\mathrm{ONFH}(\mathrm{n}=423$; 342 male and 81 female; age, $47.0 \pm 14.0$ years) and unrelated control subjects $(n=348 ; 298$ male and 50 female; age, $51.9 \pm 10.7$ years) were recruited in the present study. All individuals were consecutively enrolled at Kyungpook National University Hospital (Daegu, Korea) between 2002 and 2006, and provided informed consent. The present study was approved 
Table I. Sequences of primer and Taqman probes for genotyping of F5 single nucleotide polymorphisms

\begin{tabular}{lll}
\hline TaqMan $^{\circledR}$ genotyping assays & Probes (ABI) & \multicolumn{1}{c}{ Context Sequence (VIC/FAM) } \\
\hline rs6028 & C_8919464_20 & TTTCAGGCTTACCTGAAATGGTAGA(C/T)T \\
rs6029 & GTGGTTTTCTTTCTTAAAATATG \\
rs6022 & C_11975262_10 & GAGCCACAGCGTCGTCCATCTTCTC(C/T)G \\
rs6032 & C_11975261_10 & CAGGGAATGTGTGGTCAAGGTAAG \\
& AGATAAGCAGGGGCCCAATCAGCCC(A/C)G \\
& C_8919441_30 & AGTTGAAATCCTCGATCAGATTTT \\
& & AGTAACAGATCACTAGGAGGGTCCT(C/T)C \\
\end{tabular}

\begin{tabular}{lcl}
\hline Custom Taqman ${ }^{\circledR}$ genotyping assays & Primer, Probe & Sequences \\
\hline rs6672595 & Forward & GTGGTGTCACTACTGCAGTCA \\
& Reverse & TGAGAAGGGTTTGGCTGTGATTTT \\
rs6672595 & VIC & TCCTACGTGTATACTACC \\
& FAM & TCCTACATGTATACTACC \\
& Forward & GCCTTTGACCTCTTGCTTAAAAATGT \\
& Reverse & CCAATCCTCACTTCCAGTCCAAA \\
& FAM & ACTTGGTGATGAAATC \\
\hline
\end{tabular}

by the ethics committee of Kyungpook National University Hospital (Daegu, Korea). The patients were diagnosed using anteroposterior and lateral pelvic radiographs (Innovision-SH; DK, Seoul, Korea), and magnetic resonance images (Signa 1.5 Excite; GE Healthcare, Little Chalfont, UK). The patients with available genotype data were sub-grouped, according to etiological factors (17) into alcohol-induced (206 cases), steroid-induced (77 cases) and idiopathic (140 cases) ON groups. The controls were defined by a lack of hip pain and by the absence of any lesion with a sclerotic margin or subchondral collapse consistent with ONFH in the anteroposterior and frog leg lateral pelvic radiographs. Any relations of the patients were excluded from the control group.

Sequencing analysis of the human F5 gene. All 25 exons of the $F 5$ gene and their boundaries, including the promoter region $(\sim 1.5 \mathrm{~kb})$, were sequenced to identify single nucleotide polymorphisms (SNPs) in the 24 Korean DNA samples using a MegaBace1000 DNA analyzer (GE Healthcare) with a DYEnamic ET Dye terminator kit (GE Healthcare). The primer 3 online primer design tool (http://biotools.umassmed. edu/bioapps/primer3_www.cgi) was used to design 35 primer sets of the F5 gene for PCR amplification and sequencing analysis, based on GenBank (http://www.ncbi.nlm.nih.gov/ genbank/) sequences (Reference genome sequence for $F 5$; NT_004487.18). The sequence variants were confirmed using chromatograms.

Genotyping. Genomic DNA was isolated from the peripheral blood of each individual using a FlexiGene DNA kit (Qiagen, Valencia, CA, USA). The DNA concentration was determined using a Nanodrop 2000 spectrophotometer (Thermo Fisher Scientific, Waltham, MA USA). The genotype was determined using a TaqMan ${ }^{\mathrm{TM}}$ fluorogenic 5'-nuclease assay with pre-designed or custom TaqMan primer/probe sets (Applied Biosystems, Foster City, CA, USA). The primer and probe sequences are indicated in Table I. One allelic probe was labeled with 6-carboxyfluorescein $(\text { FAM })^{\mathrm{TM}}$ dye and the other was labeled with fluorescent $\mathrm{VIC}^{\circledR}$ dye. Genotyping was performed using TaqMan Universal master mix (Applied Biosystems) with PCR primer concentrations of $900 \mathrm{nM}$ and TaqMan MGB probe concentrations of $200 \mathrm{nM}$ and ABI 7500 real-time PCR system (Applied Biosystems). The final reaction volume for PCR was $10 \mu \mathrm{l}$ containing $20 \mathrm{ng}$ of genomic DNA. The thermal cycle conditions were as follows: $95^{\circ} \mathrm{C}$ for $10 \mathrm{~min}$, followed by 40 cycles at $95^{\circ} \mathrm{C}$ for $15 \mathrm{sec}$ and at $60^{\circ} \mathrm{C}$ for $1 \mathrm{~min}$ as previously described (18). The fluorescence data files from each plate were collected and analyzed using automated allele-calling software (SDS 2.2; Applied Biosystems). Quality control of the genotyping was performed on $10 \%$ of the samples by examining duplicates (rate of concordance in duplicates $>99 \%$ ).

Statistical analysis. The Hardy-Weinberg equilibrium (HWE) was used to determine significant deviation of the genotypic frequency from each SNP using a $\chi^{2}$ test. Statistical significance was determined using the P-values obtained from the logistical regression analysis, controlling for age and gender as covariates using three alternative models (co-dominant, dominant and recessive). To assess the risk of the phenotypes, odds ratios and 95\% confidence intervals were also estimated using a logistic regression procedure. The linkage disequilibrium (LD) between the loci was measured using the absolute value of Lewontin's D' (ID'l) (19).

The haplotype structures and frequencies were estimated from the genotyped data within the LD block using Haploview 3.32 (http://www.broad.mit.edu/mpg/haploview/), which 
Table II. Clinical profiles of patients with osteonecrosis of the femoral head and healthy controls.

\begin{tabular}{lccccc}
\hline & & \multicolumn{3}{c}{ Patients } \\
\cline { 3 - 6 } Characteristic & $\begin{array}{c}\text { Control } \\
(\mathrm{n}=348)\end{array}$ & $\begin{array}{c}\text { Total } \\
(\mathrm{n}=423)\end{array}$ & $\begin{array}{c}\text { Idiopathic } \\
(\mathrm{n}=140)\end{array}$ & $\begin{array}{c}\text { Alcohol-induced } \\
(\mathrm{n}=206)\end{array}$ & $\begin{array}{c}\text { Steroid-induced } \\
(\mathrm{n}=77)\end{array}$ \\
\hline Age (mean $\pm \mathrm{SD})^{\mathrm{a}}$ & $51.9 \pm 10.7$ & $47.0 \pm 14.0$ & $43.9 \pm 15.2$ & $50.6 \pm 12.5$ & $42.9 \pm 13.4$ \\
Gender (male/female) & $298 / 50$ & $342 / 81$ & $91 / 49$ & $198 / 8$ & $53 / 24$ \\
Involvement (uni/bilateral) & 0 & $182 / 241$ & $80 / 60$ & $74 / 132$ & $28 / 49$ \\
BMI $\left(\mathrm{kg} / \mathrm{m}^{2} ;\right.$ mean $\left.\pm \mathrm{SE}\right)$ & $23.8 \pm 0.17$ & $24.1 \pm 0.63$ & $23.4 \pm 0.38$ & $24.5 \pm 0.89$ & $22.9 / 0.53$ \\
\hline
\end{tabular}

${ }^{a} \mathrm{P}<0.05$ between total patients and controls. SD, standard deviation; SE, standard error; BMI, body mass index.

A

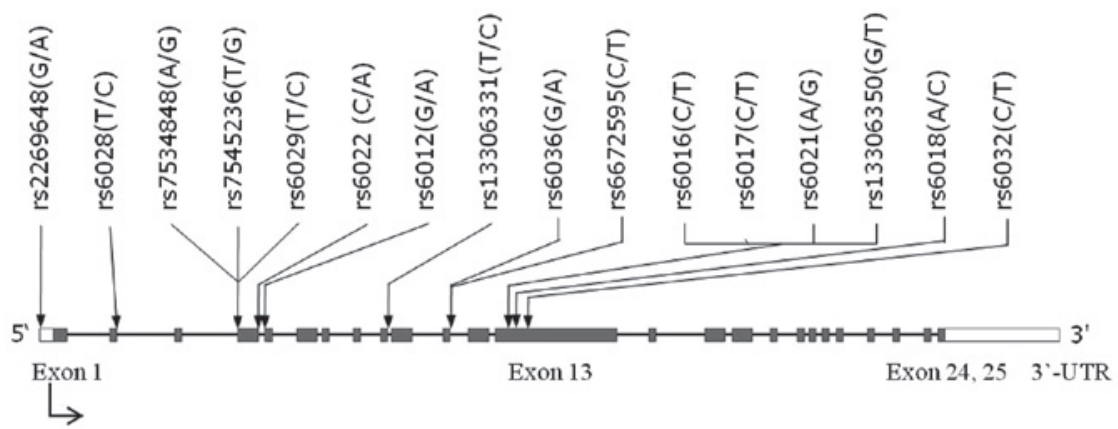

B

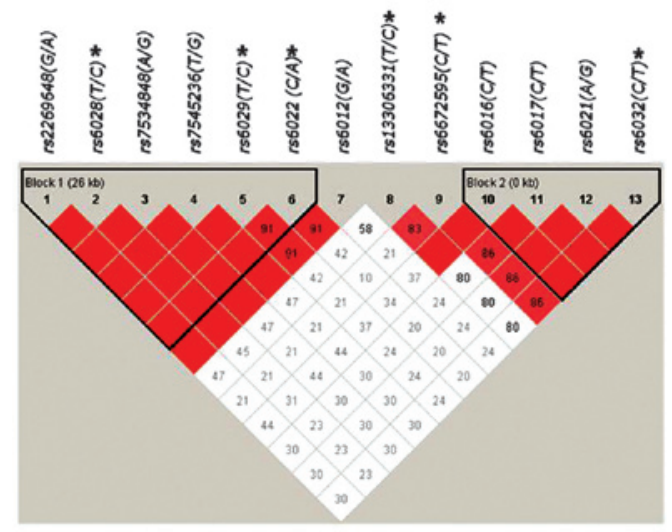

C

\begin{tabular}{|c|c|c|c|c|}
\hline Haplotype & $\begin{array}{l}\stackrel{0}{~} \\
\stackrel{్}{0} \\
\end{array}$ & $\begin{array}{l}\text { స్రి } \\
\text { ర్ర్ర }\end{array}$ & $\begin{array}{l}\text { స్ } \\
\text { ర్ } \\
\end{array}$ & Frequency \\
\hline HAP 1 & $T$ & $T$ & $A$ & 0.581 \\
\hline HAP 2 & $C$ & C & $C$ & 0.268 \\
\hline HAP 3 & $T$ & C & C & 0.150 \\
\hline
\end{tabular}

Figure 1. Map of the $F 5$ gene on chromosome 1q23 and its haplotypes. " indicates that the SNPs, which were genotyped in the population assessed in the present study. (A) Polymorphisms of the F5 gene. Coding exons are indicated by gray blocks and the 5' and 3' untranslated regions are indicated by white blocks. (B) Linkage disequilibrium coefficients $\left(\left|D^{\prime}\right|\right)$ among the identified SNPs. (C) Haplotypes of $F 5$ in Block 1. SNP, single nucleotide polymorphism; UTR, untranslated region; $F 5$, factor $\mathrm{V}$.

estimates haplotypes using an accelerated expectation-maximization (EM) algorithm (20). Fisher's exact or $\chi^{2}$ test was used to compare the frequency of discrete variables between the controls and the patients. Continuous variables were compared using Student's t-test or analysis of variance. Statistical analyses were performed using SAS 9.1 software (SAS Institute Inc., Cary, NC, USA) and $\mathrm{P}<0.05$ was considered to indicate a statistically significant difference.

\section{Results}

A total of 423 unrelated patients with ONFH and 348 control individuals were recruited for investigation in the present study. The clinical characteristics of the controls and patients are summarized in Table II. The data revealed no significant differences between the patients and the controls, with the exception of the mean age $(\mathrm{P}<0.05)$. Direct DNA sequencing of 24 individuals revealed 16 SNPs within the exons and flanking regions of $F 5$, including a 1.5 -kb promoter region, 10 in the exons, five in the exon-intron boundary and one in the promoter region (Fig. 1A). The frequencies of the identified SNPs in the Korean population are shown in Table III. Of the SNPs, rs6036, rs13306350 and rs6018 were excluded from further analysis, as they did not fulfill the criteria of a call rate $>95.0$, minor allele frequency $>0.05$ and HWE $>0.01$. Pair-wise comparisons among the 13 SNPs revealed two haplotype blocks, with each 


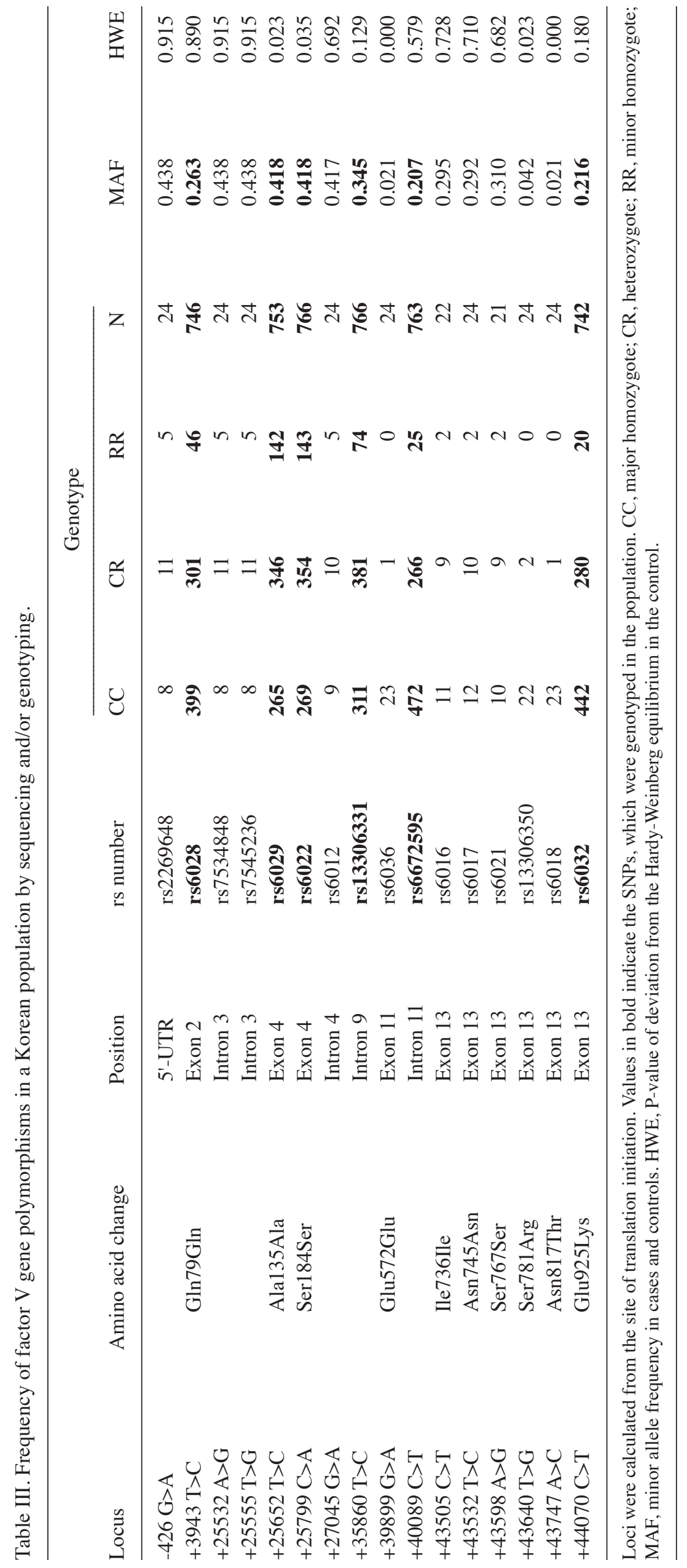




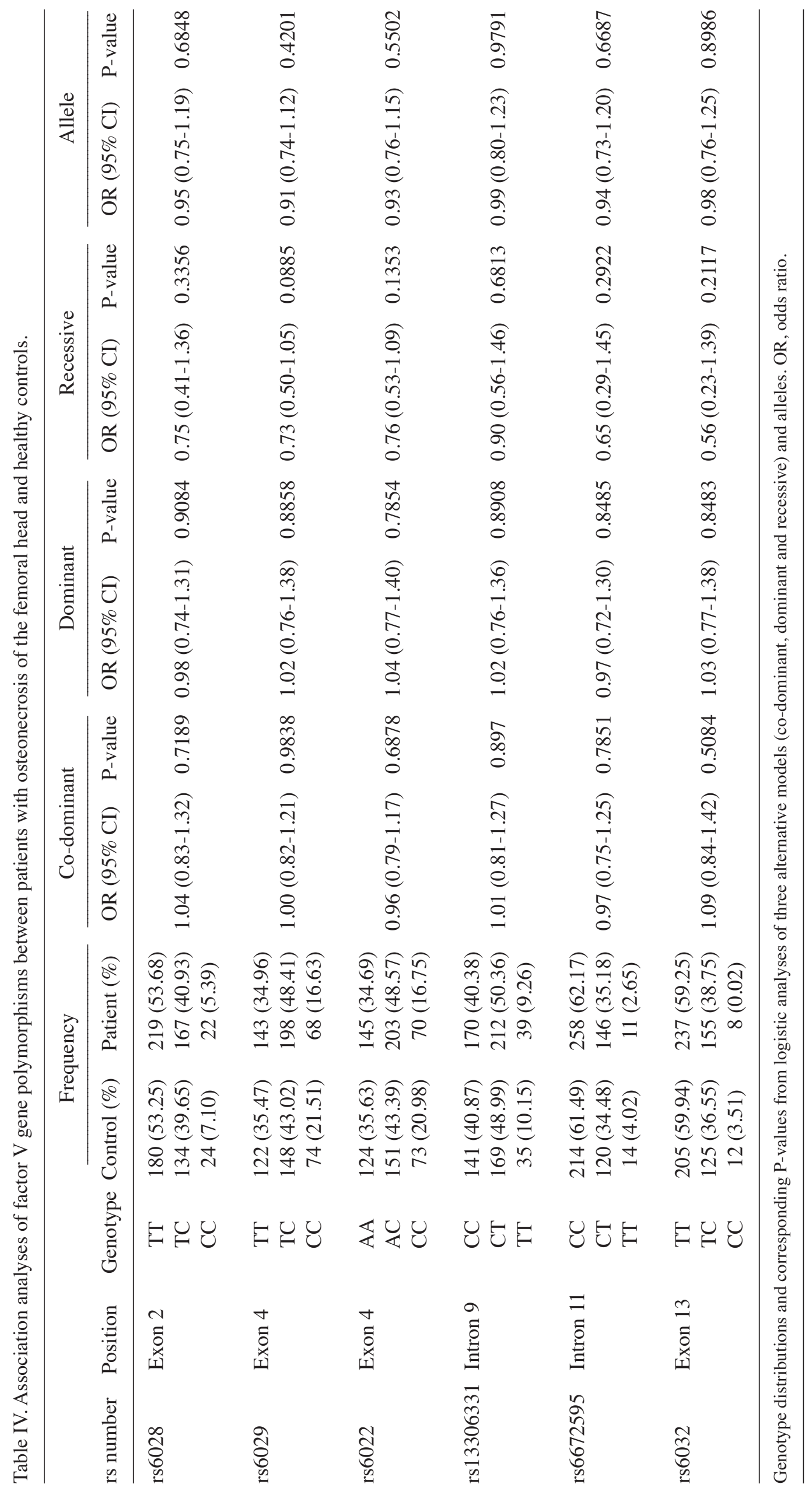


block exhibiting a marked LD spine (Fig. 1B). From these polymorphisms, six were selected for larger-scale genotyping by considering their location, allele frequencies and LD coefficients among polymorphisms (Table III), and were genotyped for association analysis in the 423 patients and 348 controls. Logistic regression analysis in all analysis models (co-dominant, dominant and recessive) revealed no significant association between the individual F5 SNPs and the risk of ONFH (Table IV).

Haplotype frequencies were estimated from the genotyped SNP data within LD block 1 using the EM algorithm. This revealed three common haplotypes (frequency $>0.05$ ) accounting for $>99 \%$ of the observed haplotypes (Fig. 1C), which were used for subsequent association analysis. No significant association was observed between the haplotypes and the risk of ONFH (Table V).

\section{Discussion}

Among the pathogenic mechanisms of ONFH that have been suggested to date, a vascular hypothesis is considered to be the most compelling. It suggests that, if thrombosis occurs, it is followed by a sequential process of blood flow obstruction, increased venous pressure, impaired arterial flow, osseous hypoxia and bone death $(11,21)$, which appear to be important in the development of ONFH. Previous studies have claimed that thrombophilia due to inherited defects, including protein $\mathrm{C}$ deficiency, protein $\mathrm{S}$ deficiency or activated protein $\mathrm{C}$ resistance, as a result of the $F 5$ Leiden mutation, may result in venous occlusion of the femoral head, leading to ONFH in adults or Legg-Calve-Perthes disease in children $(22,23)$. F5 Leiden generates coagulation $F 5$, which is degraded less effectively by activated protein $\mathrm{C}$, resulting in a hypercoagulable state.

Although an increased tendency for intravascular coagulation has been suggested as a pathogenic mechanism of ONFH, the association between genetic predisposition and thrombotic tendency may differ between ethnic groups. A number of previous studies have reported that the F5 Leiden mutation (G1691A; Arg506Gln) increases the risk of primary ON $(6,11,12)$, however other studies have failed to observe these associations $(24,25)$. Additionally, neither F5 Leiden, nor the prothrombin G20210A mutation have been identified in the Korean population $(14,16)$.

Although the $F 5$ Leiden mutation is not observed in Koreans, it is likely that the $F 5$ gene is important with respect to ONFH. Therefore, the present study aimed to identify novel common SNPs in the Korean population and to perform an association analysis. In 24 samples, $>90 \%$ of common SNPs with a frequency of $>0.05$ were expected (26). Among the 16 SNPs identified, 6 SNPs were genotyped. Comparison between the ONFH patients and control subjects revealed no significant differences in the distribution of the $F 5$ genotypes and haplotypes, suggesting that $F 5$ polymorphisms are not involved in susceptibility to ONFH in the Korean population.

SNP markers provide the primary data for population investigations. At present, $>10,000,000$ SNPs have been identified in the human genome. A large portion of these SNPs have a frequency $<5 \%$ and are, therefore, private or common in only a single population. The fixation index $\left(\mathrm{F}_{\mathrm{ST}}\right)$ is a measure of population differentiation due to genetic structure. Previous data suggested the population exhibiting the highest level of differentiation worldwide as the Amerindians $\left(\mathrm{F}_{\mathrm{ST}} ; 0.295\right)$, whereas no 
difference was observed among populations from East Asian $\left(\mathrm{F}_{\mathrm{ST}} ; 0.01\right)$, including Koreans, Japanese and Chinese populations $(27,28)$. The F5 Leiden (G1691A) allele frequency among European populations and American population of European descent is high (2-8\%) $(29,30)$, whereas those among native populations of the Far East or Africa are markedly lower or even absent (30,31). F5 Leiden and prothrombin G20210A mutations, which have been associated with ONFH in Caucasian or other population, were not observed in previous investigations of Korean populations (14-16), nor were they observed in the population in present study.

As described above, previous studies have suggested that thrombotic and fibrinolytic disorders may be etiological causes of ONFH $(5,32)$. However, in one small case-control investigation of a Korean population, no significant differences were observed in the levels of several thrombotic factors, including protein $\mathrm{C}$ and $\mathrm{S}$ activity, antithrombin and anticardiolipin antibody, and fibrinolytic factors, including tissue plasminogen activator and plasminogen activator inhibitor-1, and the data failed to confirm an etiological role for thrombotic and fibrinolytic disorders in East Asian patients with ONFH (13). A possible explanation for the conflicting results between populations may lie in geographic and ethnic differences in the prevalence of disease and/or associated SNPs.

In conclusion, the present study performed direct sequencing to detect polymorphisms, and case-control association analyses in patients with ONFH and normal control individuals using six selected SNPs of the $F 5$ gene. No evidence was obtained to support an association of $F 5$ genetic polymorphisms with $\mathrm{ONFH}$ haplotypes. Although it is important in Caucasian individuals, the results of the present study suggested that coagulation F5 is not a genetic risk factor for ONFH in the Korean population. Further investigations using larger sample sizes and detailed coagulation profiling are necessary to fully assess the significance of this gene in ONFH.

\section{Acknowledgements}

This study was supported by a grant from the Korea Health Technology R\&D Project through the Korea Health Industry Development Institute, funded by the Ministry of Health \& Welfare, Republic of Korea (grant no. HI15C0001).

\section{References}

1. Assouline-Dayan Y, Chang C, Greenspan A, Shoenfeld Y and Gershwin ME: Pathogenesis and natural history of osteonecrosis. Semin Arthritis Rheum 32: 94-124, 2002.

2. Fisher DE: The role of fat embolism in the etiology of corticosteroid-induced avascular necrosis: clinical and experimental results. Clin Orthop Relat Res 130: 68-80, 1978.

3. Wang Y, Li Y, Mao K, Li J, Cui Q and Wang GJ: Alcohol-induced adipogenesis in bone and marrow: a possible mechanism for osteonecrosis. Clin Orthop Relat Res 410: 213-224, 2003.

4. Abu-Shakra M, Buskila D and Shoenfeld Y: Osteonecrosis in patients with SLE. Clin Rev Allergy Immunol 25: 13-24, 2003.

5. Jones LC, Mont MA, Le TB, et al: Procoagulants and osteonecrosis. J Rheumatol 30: 783-791, 2003.

6. Bjorkman A, Burtscher IM, Svensson PJ, Hillarp A, Besjakov J and Benoni G: Factor V Leiden and the prothrombin 20210A gene mutation and osteonecrosis of the knee. Arch Orthop Trauma Surg 125: 51-55, 2005.

7. Zalavras CG, Vartholomatos G, Dokou E and Malizos KN: Factor $\mathrm{V}$ Leiden and prothrombin gene mutations in femoral head osteonecrosis. Thromb Haemost 87: 1079-1080, 2002.
8. Gohel A, McCarthy MB and Gronowicz G: Estrogen prevents glucocorticoid-induced apoptosis in osteoblasts in vivo and in vitro. Endocrinology 140: 5339-5347, 1999.

9. Kawai K, Tamaki A and Hirohata K: Steroid-induced accumulation of lipid in the osteocytes of the rabbit femoral head. A histochemical and electron microscopic study. J Bone Joint Surg Am 67: 755-763, 1985.

10. Kerachian MA, Harvey EJ, Cournoyer D, Chow TY and Séguin C: Avascular necrosis of the femoral head: vascular hypotheses. Endothelium 13: 237-244, 2006.

11. Zalavras CG, Vartholomatos G, Dokou E and Malizos KN: Genetic background of osteonecrosis: associated with thrombophilic mutations? Clin Orthop Relat Res 422: 251-255, 2004.

12. Bjorkman A, Svensson PJ, Hillarp A, Burtscher IM, Runow A and Benoni G: Factor V leiden and prothrombin gene mutation: risk factors for osteonecrosis of the femoral head in adults. Clin Orthop Relat Res 425: 168-172, 2004.

13. Lee JS, Koo KH, Ha YC, et al: Role of thrombotic and fibrinolytic disorders in osteonecrosis of the femoral head. Clin Orthop Relat Res 417: 270-276, 2003.

14. Chang JD, Hur M, Lee SS, Yoo JH and Lee KM: Genetic background of nontraumatic osteonecrosis of the femoral head in the Korean population. Clin Orthop Relat Res 466: 1041-1046, 2008.

15. Hessner MJ, Luhm RA, Pearson SL, Endean DJ, Friedman KD and Montgomery RR: Prevalence of prothrombin G20210A, F5 G1691A (Leiden) and methylenetetrahydrofolate reductase (MTHFR) C677T in seven different populations determined by multiplex allele-specific PCR. Thromb Haemost 81: 733-738, 1999.

16. Kim SY, Suh JS, Park EK, et al: Factor V Leiden gene mutation in femoral head osteonecrosis. J Korean Ortho Res Soc 6: 259-264, 2003.

17. Hong JM, Kim TH, Chae SC, et al: Association study of hypoxia inducible factor $1 \alpha(\mathrm{HIF} 1 \alpha)$ with osteonecrosis of femoral head in a Korean population. Osteoarthritis Cartilage 15: 688-694, 2007.

18. Lee HJ, Choi SJ, Hong JM, et al: Association of a polymorphism in the intron 7 of the SREBF1 gene with osteonecrosis of the femoral head in Koreans. Ann Hum Genet 73: 34-41, 2009.

19. Hedrick PW: Gametic disequilibrium measures: proceed with caution. Genetics 117: 331-341, 1987.

20. Barrett JC, Fry B, Maller J and Daly MJ: Haploview: analysis and visualization of LD and haplotype maps. Bioinformatics 21: 263-265, 2005.

21. Glueck CJ, Freiberg R, Tracy T, Stroop D and Wang P: Thrombophilia and hypofibrinolysis: pathophysiologies of osteonecrosis. Clin Orthop Relat Res 334: 43-56, 1997.

22. Glueck CJ, Brandt G, Gruppo R, et al: Resistance to activated protein $\mathrm{C}$ and Legg-Perthes disease. Clin Orthop Relat Res 338: 139-152, 1997.

23. Gruppo R, Glueck CJ, Wall E, Roy D and Wang P: Legg-Perthes disease in three siblings, two heterozygous and one homozygous for the F5 Leiden mutation. J Pediatr 132: 885-888, 1998.

24. Glueck CJ, Fontaine RN, Gruppo R, et al: The plasminogen activator inhibitor-1 gene, hypofibrinolysis and osteonecrosis. Clin Orthop Relat Res 366: 133-146, 1999.

25. Celik A, Tekis D, Saglam F, et al: Association of corticosteroids and F5, prothrombin and MTHFR gene mutations with avascular osteonecrosis in renal allograft recipients. Transplant Proc 38: 512-516, 2006.

26. Eberle MA and Kruglyak L: An analysis of strategies for discovery of single-nucleotide polymorphisms. Genet Epidemiol 19 (Suppl 1): 29-35, 2000.

27. Adachi N,ShinodaK, Umetsu K and Matsumura H: Mitochondrial DNA analysis of Jomon skeletons from the Funadomari site, Hokkaido and its implication for the origins of Native American. Am J Phys Anthropol 138: 255-265, 2009.

28. Jung J, Kang H, Cho YS, et al: Gene flow between the Korean peninsula and its neighboring countries. PloS one 5: e11855, 2010.

29. Bowen DJ, Bowley S, John M and Collins PW: Factor V Leiden (G1691A), the prothrombin 3'-untranslated region variant (G20210A) and thermolabile methylenetetrahydrofolate reductase (C677T): a single genetic test genotypes all three loci-determination of frequencies in the $\mathrm{S}$. Wales population of the UK. Thromb Haemost 79: 949-954, 1998.

30. Rees DC, Cox M and Clegg JB: World distribution of $F 5$ Leiden. Lancet 346: 1133-1134, 1995.

31. Pepe G, Rickards O, Vanegas OC, et al: Prevalence of F5 Leiden mutation in non-European populations. Thromb Haemost 77: 329-331, 1997.

32. Glueck CJ, Freiberg RA and Wang P: Heritable thrombophilia-hypofibrinolysis and osteonecrosis of the femoral head. Clin Orthop Relat Res 466: 1034-1040, 2008. 